\title{
An overview of traffic congestion detection and classification techniques in VANET
}

\author{
Nurshahrily Idura Ramli, Mohd Izani Mohamed Rawi \\ Faculty of Computer and Mathematical Sciences, Universiti Teknologi MARA (UiTM), Malaysia
}

\begin{tabular}{l} 
Article Info \\
\hline Article history: \\
Received Feb 4, 2020 \\
Revised Apr 5, 2020 \\
Accepted Apr 19, 2020 \\
\hline
\end{tabular}

\section{Keywords:}

Qualitative analysis

Simulated mobility environment

Traffic congestion classification

Traffic congestion detection

Vehicular ad-hoc network

\begin{abstract}
Vehicular traffic congestion has been and still is a major problem for many countries and knowledge about the traffic condition is important in order to schedule, plan and avoid traffic congestion. With recent development in technology, various efforts and methods are proposed in mitigating traffic congestion. Vehicular Ad-hoc NETwork (VANET) is very much in the hype in addressing this issue due to its capabilities and adaptation to scalability, highly dynamic topology as well as cooperative communication. A popular focus is in detecting and classisying traffic congestion which presents various techniques and methodologies. This paper presents an overview of traffic congestion detection and classification methods of various related techniques in VANET, organized from the research perspective. Qualitative analysis is presented to classify these strategies in its system architecture, detection and classification methods, as well as its simulated mobility environment and simulation tools used. The analysis is useful in understanding all the techniques and methods applied in resolving this issue in the research domain.
\end{abstract}

Copyright $@ 2020$ Institute of Advanced Engineering and Science. All rights reserved.

\section{Corresponding Author:}

Nurshahrily Idura Ramli,

Faculty of Computer and Mathematical Sciences,

Universiti Teknologi MARA (UiTM), Shah Alam, Selangor, Malaysia.

Email: idura@tmsk.uitm.edu.my

\section{INTRODUCTION}

For the last decades, traffic congestion has caused significant impact in modern society. Despite the measures taken to reduce the impact it bestowed, traffic congestion is affecting the environment, health, and crippling the economy of many cities around the world in so many different levels. Generally, there are many factors that can cause traffic to congest, and it could be caused by either recurring factors such as insufficient capacity, unrestrained demand or ineffective management of capacity or caused by non-recurring factors such as incidents, construction work zones, bad weather or emergencies [1]. To this effect, the world is witnessing the rise in transportation as well as urban technology researches in addressing this issue. Some are focusing on detecting and classifying traffic congestion in order to provide timely and accurate information to vehicular drivers and transport authorities to take action. While many researches are utilizing on image and video analytics such as through satellites views, surveillance cameras, on-line images, there's a hype in researches utilizing VANET.

Vehicular communications have been extensively researched with the aim of enabling vehicles to exchange information among themselves, also with the infrastructure. This enables the collection of vehicles' traffic data that then ratifies the road traffic characteristics, including speed, density, flow, and travel time. With the data collected, many researches have adopted various methodologies to among others; control congestion [2,3], provide various applications such as safety alerts, resolving security issues, and many more. A particular focus includes mathematical and statistical algorithms, fuzzy logic, neural network, classifiers as well as utilizing the routing mechanism in VANET networks in detecting and classifying traffic congestion.

Classification of the severity of congestion levels is important to define the state of traffic congestion levels in order to avoid and mitigate traffic congestion. This classification and other traffic information approach often rely 
on a traffic management system such as ITS that necessitates the overhead of space, transmission, and delay in its database processing. The dependency of a centralized system such as this would further lead to disseminating traffic alerts and information that may be irrelevant at a point of time of need; where rerouting is no longer possible. This would avert the driver to avoid traffic congestion, and thus boosting the congestion escalation. The premise of this overview is to analyze the techniques implemented in traffic congestion detection and classification in VANET. The contribution of this survey are: 1) An overview of the VANET system architecture; 2) A qualitative analysis that includes the chronological development of the methods, VANET system architecture, traffic congestion detection strategies, traffic congestion classification methods, mobility model scenario and the simulation tools used; 3) Diverse approaches to traffic congestion detection; 4) Diverse approaches to traffic congestion classification and 5) A review of the selection of simulation models and tools used for simulating VANET environment.

\section{VANET SYSTEM ARCHITECTURE}

VANETs is considered a key component of the Intelligent Transportation System (ITS) architecture [4, 5]. The communication specifications for VANET is defined by IEEE802.11p and IEEE1609 represent the most mature set of standards for wireless vehicular networks or also referred to as DSRC/WAVE networks. VANET's main system components are the Application Unit (AU), the On-Board Unit (OBU) and the Road Side Unit (RSU) [6].

VANET systems is categorized as centralized, decentralized and hybrid; it is set upon its dependency of with or without the use of fixed infrastructure and a central server [7]. Centralized systems that operates on fixed infrastructure and a central server are difficult to scale and often have issues with cost and delay while decentralized systems that run without any fixed infrastructure and a central server still needs to conforms to the limitation of the network such as communication barriers, data redundancy, bandwidth capacity, network reliability and etc [8]. Nonetheless, many are balancing both approaches with the hybrid approach, often using RSU to compensate the limitations of network connectivity, and to improve in scalability, processing, routing and disseminating information in estimating the global traffic view [9-11]. This enables the technology to support communication between vehicles through vehicle to vehicle communication (V2V) or communication of vehicles with any RSUs for vehicle to infrastructure communication (V2I) [12-14].

\section{OVERVIEW OF VEHICULAR TRAFFIC CONGESTION DETECTION AND CLASSIFICATION TECHNIQUES IN VANET}

This section presents a qualitative analysis of vehicular traffic congestion detection and classification techniques in VANET found in references. Table 1 describes the proposed qualitative analysis organized in chronological order, system architecture, traffic congestion detection strategies, traffic congestion classification methods used in VANET, while Table 2 enlists the simulation mobility environment and simulation tools used in each technique.

Table 1. Qualitative analysis of vehicular traffic congestion detection and classification techniques

\begin{tabular}{|c|c|c|c|c|c|c|c|c|c|c|c|c|}
\hline \multirow[b]{2}{*}{ Year } & \multirow{2}{*}{ 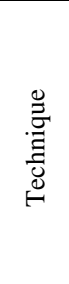 } & \multicolumn{2}{|c|}{$\begin{array}{c}\text { System } \\
\text { Architecture } \\
\text { Design }\end{array}$} & \multicolumn{5}{|c|}{ Traffic Congestion Detection Strategy } & \multicolumn{4}{|c|}{$\begin{array}{c}\text { Traffic Congestion Classification } \\
\text { Method }\end{array}$} \\
\hline & & 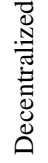 & Hybrid & $\begin{array}{l}\text { Fuzzy } \\
\text { Logic }\end{array}$ & ANN & 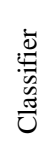 & 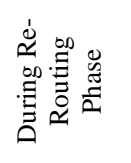 & $\frac{\tilde{d}}{\tilde{\Xi}}$ & 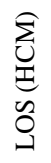 & ANN & 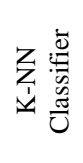 & $\stackrel{\bar{\Xi}}{\bar{\Xi}}$ \\
\hline 2012 & {$[15]$} & & $\checkmark$ & & & & $\bar{\checkmark}$ & & $\checkmark$ & & & \\
\hline 2013 & [35] & $\checkmark$ & & $\checkmark$ & & & & & $\checkmark$ & & & \\
\hline 2014 & [38] & $\checkmark$ & & & & & $\checkmark$ & & & & & $\checkmark$ \\
\hline 2014 & [20] & $\checkmark$ & & $\checkmark$ & & & & & $\checkmark$ & & & \\
\hline 2015 & [28] & $\checkmark$ & & & $\checkmark$ & & & & & $\checkmark$ & & \\
\hline 2015 & [32] & & $\checkmark$ & & & $\checkmark$ & & & $\checkmark$ & & & \\
\hline 2015 & {$[37]$} & & $\checkmark$ & & & & & $\checkmark$ & & & $\checkmark$ & \\
\hline 2015 & {$[16]$} & & $\checkmark$ & & & & $\checkmark$ & & & & $\checkmark$ & \\
\hline 2016 & [17] & $\checkmark$ & & & & & $\checkmark$ & & $\checkmark$ & & & \\
\hline 2016 & [33] & & $\checkmark$ & & & $\checkmark$ & & & $\checkmark$ & & & \\
\hline 2016 & [29] & $\checkmark$ & & & $\checkmark$ & & $\checkmark$ & & & $\checkmark$ & & \\
\hline 2016 & [30] & & $\checkmark$ & & & $\checkmark$ & & & & $\checkmark$ & & \\
\hline 2018 & [21] & & & $\checkmark$ & & & & & & & & $\checkmark$ \\
\hline 2019 & [22] & $\checkmark$ & & $\checkmark$ & & & & & & & & $\checkmark$ \\
\hline 2019 & [23] & & & $\checkmark$ & & & & & & & & $\checkmark$ \\
\hline
\end{tabular}

Indonesian J Elec Eng \& Comp Sci, Vol. 20, No. 1, October 2020 : 437 - 444 
Table 2. Simulation tools and mobility environment used in VANET simulations

\begin{tabular}{|c|c|c|c|c|c|c|c|c|c|c|c|}
\hline \multirow[b]{2}{*}{$\begin{array}{l}\stackrel{0}{\tilde{J}} \\
\stackrel{\Xi}{\Xi} \\
\stackrel{e}{\oplus}\end{array}$} & \multicolumn{3}{|c|}{ Simulation Mobility Environment } & \multicolumn{8}{|c|}{ Simulation Tools Used } \\
\hline & Urban & $\begin{array}{l}\text { High- } \\
\text { Way }\end{array}$ & Simulated Scenario & SUMO & $\begin{array}{l}+ \\
+ \\
\stackrel{ \pm}{ \pm} \\
\sum_{0}^{\prime}\end{array}$ & $\mathrm{ns} 2$ & $\mathrm{~ns} 3$ & 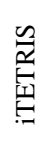 & $\stackrel{a}{a}$ & $\sum_{\text {䛼 }}$ & $\begin{array}{l}0 \\
80 \\
0 \\
9 \\
\stackrel{1}{0} \\
\text { Z }\end{array}$ \\
\hline [15] & $\sqrt{ }$ & & Newark \& Brooklyn & $\sqrt{ }$ & & & & & & & \\
\hline$[35]$ & & $\checkmark$ & SUMO Highway Scenario & $\checkmark$ & & & $\checkmark$ & $\checkmark$ & & & \\
\hline [38] & $\checkmark$ & & Madrid & $\checkmark$ & $\checkmark$ & & & & & & \\
\hline [20] & $\checkmark$ & & Manhattan & $\checkmark$ & $\checkmark$ & & & & $\checkmark$ & & \\
\hline [28] & $\checkmark$ & & Manhattan & $\checkmark$ & $\checkmark$ & & & & $\checkmark$ & & \\
\hline [32] & $\checkmark$ & & Manhattan & $\checkmark$ & & & & & & $\checkmark$ & \\
\hline [37] & $\checkmark$ & & Manhattan & & & $\checkmark$ & & & & & \\
\hline [16] & $\checkmark$ & & TAPAS Cologne, Germany & $\checkmark$ & $\checkmark$ & & & & $\checkmark$ & $\checkmark$ & \\
\hline [17] & $\checkmark$ & & Manhattan & $\checkmark$ & $\checkmark$ & & & & $\checkmark$ & & \\
\hline [33] & $\checkmark$ & & Manhattan & $\checkmark$ & & & & & & $\checkmark$ & \\
\hline [29] & $\checkmark$ & $\checkmark$ & $\begin{array}{c}\text { Manhattan \& Dom Pedro I } \\
\text { Freeway }\end{array}$ & $\checkmark$ & $\checkmark$ & & & & $\checkmark$ & $\checkmark$ & \\
\hline [30] & $\checkmark$ & & Manhattan & $\checkmark$ & $\checkmark$ & & & & $\checkmark$ & $\checkmark$ & \\
\hline [21] & $\checkmark$ & & Beijing & & & & $\checkmark$ & & & & \\
\hline [22] & & & a two-way six-lane road & $\checkmark$ & $\checkmark$ & & & & $\checkmark$ & & \\
\hline [23] & & & Simulation of one 2-way road & & & & & & & & $\checkmark$ \\
\hline
\end{tabular}

\subsection{Traffic congestion detection}

The common traffic congestion detection strategies gathered from literature are being classified in four different strategies; 1) re-routing phase 2) fuzzy logic 3) artificial neural network and 4) classifiers.

\subsubsection{During the re-routing phase}

Traffic detection during re-routing phase is the strategy used in Dynamic Shortest Path (DSP), Random k Shortest Paths (RkSP) and Entropy Balanced k Shortest Paths (EBkSP) by Pan et al. [15], Brennand et al. [16] and FASTER [17]. DSP, RkSP, and EBkSP are three traffic re-routing strategies that operate through V2I communication whereby the vehicle geographic position, speed, and direction are acquired to detect traffic congestion. In the first data collection and representation phase, a directed graph is used to represent the network in which intersections are represented by the vertices, the edges signify the road segments, and weights are the average travel time. From this representation, signs of congestion are detected through periodic checkings. The detection, however, does not happen in real time, rather in the next re-routing phase.

Similarly in [16], based on a set of distributed RSUs covering an urban area, traffic congestion is detected when it occurs within the RSU's communication coverage. This strategy is solely dependent on V2I communication in optimizing the traffic flow. Data collection from every vehicle within the communication range consisting of the vehicle's ID, speed, direction, position and travel time through the route are gathered at the RSU via LTE or 3G single-hop, long-range communication [18]. FASTER differ from the above techniques in its communication design, in which FASTER only relies on V2V communication instead of V2I using RSUs. Instead of aggregating data within the RSU's communication range, FASTER creates smaller areas called districts in order to identify the traffic condition in those districts. Periodically, each vehicle will convey their individual traffic information through beacons to neighbouring vehicles within the same district. An overall traffic knowledge is achieved in the dissemination of each district's knowledge to other districts as in the exchanges of information by the vehicles.

\subsubsection{Fuzzy logic}

Fuzzy logic is also a popular mechanism used in VANET. Besides been utilized in detecting traffic congestion as in COTEC [19], CARTIM [20], Rui et.al [21], and most recently Wang et.al [22] and TraD-VANET [23] it is also implemented in localization, clustering, beaconing dissemination, aggregation, and other processes in VANET [24-27]. The fuzzy mechanism detects traffic congestion based on vehicular speed and traffic density. The local traffic density is obtained through CAM messages of neighboring vehicles, which is further calculated with the number of neighboring vehicles, distance to the estimating vehicle, and the number of lanes on the road. These values are then used to classify the traffic congestion level through a fuzzy-based decision system. 


\subsubsection{Artificial neural network}

Apart from using fuzzy logic mechanism, artificial neural network (ANN) which is a bio-inspired based system is also been developed to solve different complex problems and can be used efficiently to categorize the congestion states as applied in UCONDES [28], INCIDEnT [29] and ICARUS [30]. ANN is composed of several "neurons" or elementary processing units that are connected with each other in accordance with some assigned weights. Each "neuron" takes input from a source of information and produces output with the help of transfer function [31].

A Multi-Layer Perceptron ANN is used in [17] and [30] to detect traffic congestion in urban environment which is using the vehicle speed and neighboring vehicles density as input parameters for ANN to detect and classify the level of traffic congestion. Three layers are configured; (i) two neurons to represent speed and surrounding density at the input layer; (ii) four neutrons at a hidden layer that is able to learn and classify congestion levels; and (iii) an output layer neuron representing the classification of the level of congestion on the roads. Figure 1 illustrates a basic form of an ANN topology used in detecting, identifying and classifying traffic congestion as applied in several techniques such as UCONDES.

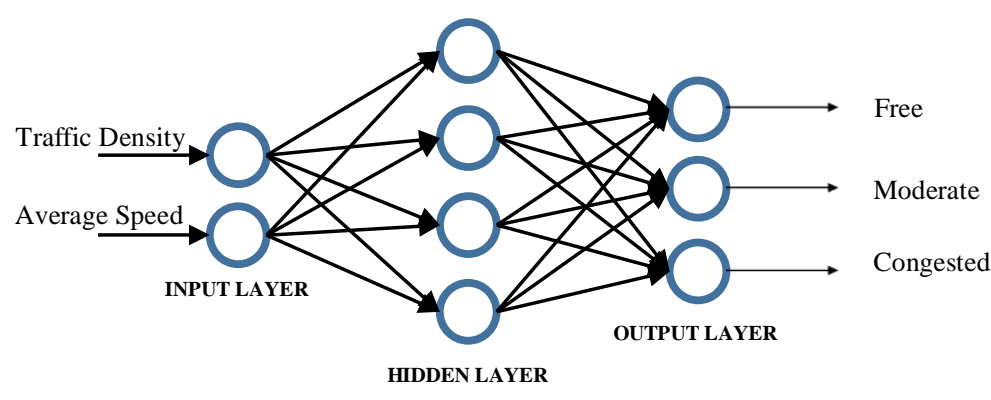

Figure 1. An example of ANN topology used in UCONDES [28]

\subsubsection{Classifiers}

SCORPION (System with COoperative Routing to improve traffic cONdition) [32] and CHIMERA (Congestion avoidance through a traffIc classification MEchanism and a Re-routing Algorithm) [33] were developed in tandem in terms of years. Both mechanisms are based on a hybrid system in utilization of the RSU which offers traffic congestion detection, traffic congestion classification and route suggestion to avoid the congestion. Traffic detection applied for both mechanisms is similar to [7] and [8], using weighted graphs. Single-hop, long-range communication adapting $4 \mathrm{G}$ and LTE is used as a communication medium in transmitting information consisting of the vehicle's ID, current position, route, and destination from vehicles to a central hub (RSU). Traffic congestion classification is fulfilled using K-Nearest Neighbor (KNN) classifier; which will be briefly described in the following section.

\subsection{Traffic congestion classification methods}

Amongst the traffic congestion classification methods utilized by the techniques in this overview are; 1) Level of Service (LOS) from the Highway Capacity Manual (HCM) 2) Artificial Neural Network 3) K-NN Classifier 4) other classification methods.

\subsubsection{Level of service (LOS) in HCM}

Highway Capacity Manual (HCM, 2000) defined LOS as "a quality measure describing operational conditions within a traffic stream, generally in terms of such service measures as speed and travel time, freedom to maneuver, traffic interruptions, and comfort and convenience."[34]. The concept of six levels of service to describe the quality of road operationsusing A to F letter scale first appeared in the 1965 HCM [34]. LOS is described in six levels of service described, ranging from LOS ' $\mathrm{A}$ ' to LOS ' $\mathrm{F}^{\prime}$. LOS A' denotes the best operating conditions of a road compared to all level of services and LOS $=F$ ' defines the worst. Table 2 defines the general LOS, that is used as a guide to classify traffic congestion in $[15,17,20,32,33,35]$, however it is important to note that this specific definition of LOS 'A' through ' $F$ ' vary by facility type [36].

\subsubsection{Artificial neural network (ANN)}

As described in in the previous section describing the Multi-Layer Perceptron ANN, upon receiving the two traffic parameters from the input layer, the neurons on the hidden layer performs computations on 
the parameters that are already assigned to some weights and passes the information to the output layer. The neurons at the output layer then classify the congestion states according to calculated weights into three categories namely High congestion, Medium congestion, and free flow subsequently distributing the information to the outside world.

\subsubsection{K-NN classifier}

The K-NN is a simple machine learning algorithm that stores all available cases and classifies data or case based on a similarity context of the aggregated data. The classification is accomplished by a majority vote of the most common of the predominant class known among its $\mathrm{k}$ nearest neighbors. It is also characterized as a lazy algorithm, defined as having less ability and is labor intensive when dealing with large datasets [22, 23]. The operation of this algorithm is based on comparing a newly received record with the training records and finding training records identical to it. All training records are stored in an $\mathrm{n}$-dimensional space, and each record with $\mathrm{n}$ attributes represents a point in the $\mathrm{n}$-dimensional space.

Upon receiving a new record, K-NN algorithm starts to find the space for the training record that is nearest to the new record, assigns this as the new record neighbors and hence predicts the class label for the new record which is similar to the identified neighbors. The algorithm defines nearest in terms of distance metrics, such as the Euclidean distance metric that could define the distance between two records. The identified $\mathrm{k}$ neighbors records are then combined, and the algorithm then assigns the classification of the most similar record or records to the new record [24]. SCORPION and CHIMERA both are using KNN classifier to classify the traffic congestion. These techniques train the algorithm based on a synthetic dataset built upon reference to the LOS in HCM.

\subsubsection{Other classification methods}

LOS in HCM and ANN is reviewed as recent and popular methods used by many techniques for traffic management in VANET. However, there are also other approaches to classifying traffic congestion. Upon the basis of traffic information received such as speed, density, number of vehicles per mile per lane, estimated travel time and direction, there are various ways that this information is manipulated into classifying the traffic congestion state. ECODE [37] classifies congestion from traffic information from Traffic Monitoring Record (TMR) generated by vehicles in specific evaluating zones, however, the mechanism or classification values were not discussed.

ABEONA [38] classifies traffic congestion based on the Three-Phase Traffic flow model. This flow model classifies traffic condition into three phases; a 'free flow', 'synchronized flow' and 'wide moving jam'. The distinction between the two congested traffic phases (synchronized and wide moving jam) is made through empirical observation in relation to spatio-temporal features of the phases. Free flow traffic is characterized by the flow of vehicles at high speed, which might be in contras with other neighboring lanes. Wide moving jams describes a condition vehicles moving in very low speeds, as low as zero at a time, and the downstream congestion front propagates upstream with a constant average velocity. The third phase, the synchronized flow defines traffic condition with moving vehicles (non-zero speed) and all congested traffic conditions which are in contrast with the wide moving jam phase [39]. There are many other classification methods that are not discussed thoroughly in this paper. This ranges from statistical approaches such as using Naïve Bayes classifiers to more complex machine learning such as Decision Tree and Random Forest to bio-mimicry methods such as Support Vector Machine (SVM) with particle swarm optimization and Hemorheology-based Traffic Congestion model [40-47]. Level of Service (LOS) in HCM as shown in Table 3.

Table 3. Level of Service (LOS) in HCM

\begin{tabular}{ccc}
\hline Level of Service & Flow Characteristics & Operating Conditions \\
\hline A & Free Flow & Low Volumes and High Speeds. \\
B & Reasonable Free Flow & Speed starting to slow down due to traffic conditions. \\
C & Stable Flow & Restrictions as far as the freedom of the drivers to choose their own speed. \\
D & Flow Approaching Instability & Drivers have limited freedom of manoeuvre. \\
E & Instable Flow & Possible brief stops. \\
F & Forced Flow & Congestion. \\
\hline
\end{tabular}

\section{SIMULATION MODELS AND TOOLS}

There are various tools providing a myriad of models in simulating VANET environments interactions be fitting the requirements of scalability and applicability, some calibration with one another [48]. As shown in Table 2, SUMO (Simulator for Urban MObility) is the most widely used traffic mobility simulation tool. SUMO supports the simulation of multimodal traffic and vehicle mobility traces to be evaluated with a network simulator on-line [49]. Network simulators listed in Table 2 are OMNeT++ 
(Objective Modular Network Testbed in C++), ns-2 (Network Simulator 2) and ns-3 (Network Simulator 3) which are most commonly used to simulate VANET environment. These network simulation tools, however, are not able to simulate road traffic. Therefore iTETRIS and VEINS (Vehicles in Network Simulation) are needed as tools to provide modular simulation platforms that integrate traffic mobility simulator and network simulator to enable wireless communication in real time road traffic simulations. iTETRIS integrates and extends SUMO and ns-3, while VEINS integrates SUMO with OMNeT++.

EMIT is a statistical model that can be imported in SUMO, specifically to measure the CO (carbon dioxide) emissions and fuel consumption of vehicles [30]. Netlogo is a multi-agent programmable modeling environment that although is recently utilized, it is not a prefered or in favor of many researches or studies in simulating VANET environment. Through the qualitative analysis in Table 2, simulation tools used by most approaches are SUMO, OMNeT++, and VEINS. SUMO is applied as the road traffic mobility generator, OMNeT++ is applied as the event-based network simulator and VEINS simulation framework is needed to bind both SUMO and OMNeT++. The calibration of these three simulators enables online bidirectional coupling in simulating real-world traffic scenarios in VANET [50]. Urban or highway are common scenarios imported to SUMO from OpenStreetMap. Figure 2 depicts the Veins architecture in simulating VANET environments.

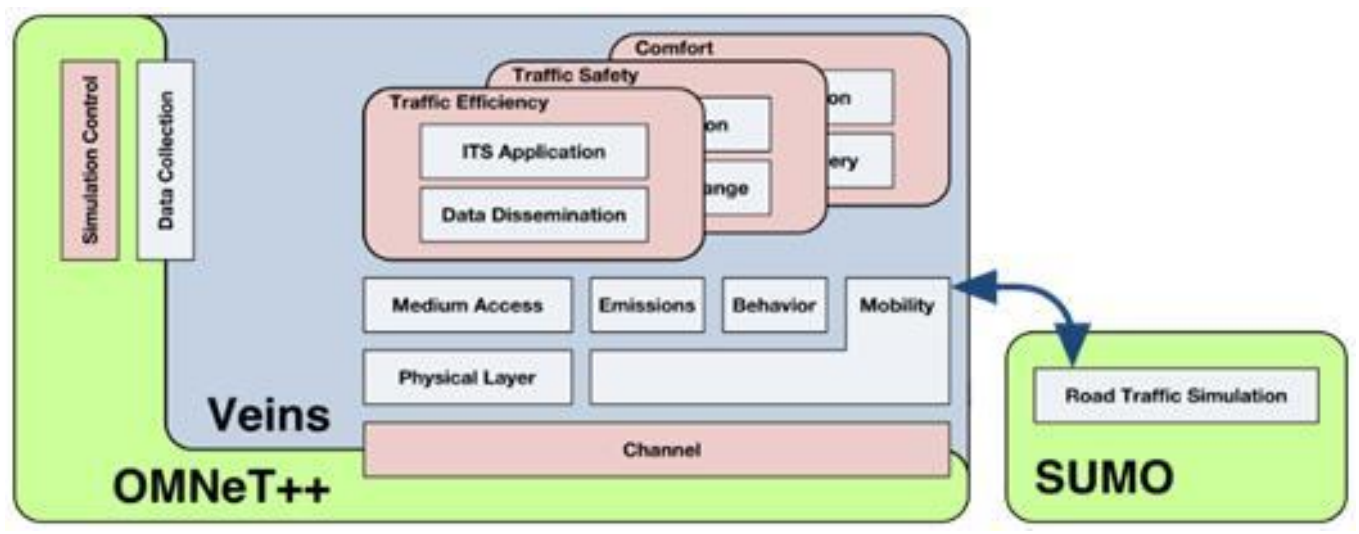

Figure 2. Veins architecture for VANET simulation

\section{CONCLUSION}

In this paper, we highlight the techniques that utilize VANET technology in the detection and classification of traffic congestion in the efforts of reducing and mitigating traffic congestion. We further provide an overview of various development of each segment in the research perspective, understanding the methodological approach, specifying its system architecture, detection strategy, and classification methods. The mobility model and simulation tools used for each technique presented in literature were also stated for reference. It is clear, through the references and qualitative analysis proposed that VANET is a well-adapted technology being utilized today in preference of its ad-hoc nature. Limitations of scalability, network connection and dynamic topology that often rises in traffic congestion detection are preferably addressed by applying a hybrid VANET system using RSU. Most techniques are classifying traffic congestion in reference to LOS in HCM and balance traffic by dispursing to other less congested routes. These strategies were developed mostly to cater the traffic issues of urban rather than the highway mobility scenario. One commonality is observed in the choice of simulation tools used by most strategies in testing, which are based on SUMO and OMNeT++. It could be concluded that there are myriad approaches and strategies in detecting and classifying traffic congestion, yet there is weak evidence of any superior or better strategy as compared to the other. This is due to the dynamics of VANET itself in allowing multiple solutions to operate in its architecture. It is interesting though to test and compare these strategies in terms of performance, scalability, and operation especially in its network communication in a fixed and similar architecture and environment, to investigate the strength and weaknesses of each approach in future.

\section{ACKNOWLEDGEMENTS}

The authors gratefully acknowledge the help of Institute of Research Management and Innovation (IRMI), Universiti Teknologi MARA (UiTM) and the Ministry of Higher Education, Malaysia in providing the Fundamental Research Grant Scheme (FRGS) (Project Number: 600-IRMI/FRGS 5/3 (203/2019) research grant. 


\section{REFERENCES}

[1] K. Nellore and G. P. Hancke, "A Survey on Urban Traffic Management System Using Wireless Sensor Networks," Sensors, vol. 16, no. 2, pp. 157-181, 2016.

[2] A. Mohanty, et al., "Traffic congestion detection in a city using clustering techniques in VANETs," Indonesian Journal of Electrical Engineering and Computer Science, vol. 13, no. 3, pp. 884-891, 2019.

[3] S. J. Elias, et al., "Congestion control in vehicular adhoc network : a survey," Indonesian Journal of Electrical Engineering and Computer Science, vol. 13, no. 3, pp. 1280-1285, 2019.

[4] O. S. Al Mushayt, et al., "Multicast routing protocol for advanced vehicular ad hoc networks," TELKOMNIKA Telecommunication Computing Electronics and Control, vol. 17, no. 3, pp. 1096-1100, 2019.

[5] T. Darwish and K. A. Bakar, "Traffic density estimation in vehicular ad hoc networks: A review," Ad Hoc Networks, vol. 24, pp. 337-351, 2015.

[6] S. ur Rehman, et al., "Vehicular Ad-Hoc Networks (VANETs) - An Overview and Challenges," Journal of Wireless Networking and Communications, vol. 3, no. 3, pp. 29-38, 2013.

[7] M. S. Kakkasageri and S. S. Manvi, "Information management in vehicular ad hoc networks: A review," Journal of Network and Computer Applications, vol. 39, no. 1, pp. 334-350, 2014.

[8] D. B. Nguyen, et al., "An Efficient Traffic Congestion Monitoring System on Internet of Vehicles," Wireless Communication and Mobile Computing, vol. 2018, pp. 1-17, 2018.

[9] S. Medetov, et al., "A decentralized approach for information dissemination in Vehicular Ad hoc Networks," Jornal of Network and Computer Applications, vol. 46, pp. 154-165, 2014.

[10] K. Gomi, "RSU Placement Method Considering Road Elements for Information Dissemination," The Sixth International Conference on Advances in Vehicular Systems, Technologies and Applications, pp. 68-73, 2017.

[11] E. Cavalcante, et al., "Roadside unit deployment for information dissemination in a VANET," Proceedings of Fourteenth Annual Conference Companion on Genetic and Evolutionary Computation, pp. 27-34, 2012.

[12] H. Moustafa and Y. Zhang, "Vehicular Networks Techniques, Standards and Applications," CRC Press Taylor \& Francis Group, An Auerbach Publication, 2009.

[13] S. Al-sultan, et al., "A comprehensive survey on vehicular Ad Hoc network," Jornal of Network anf Computer Application, vol. 37, pp. 380-392, 2014.

[14] F. Perry, et al., "Dedicated Short-Range Communications Roadside Unit Specifications," U. S. Department of Transportation, 2017.

[15] J. Pan, et al., "Proactive vehicle re-routing strategies for congestion avoidance," 2012 IEEE 8th International Conference on Distributed Computing in Sensor Systems, pp. 265-272, 2012.

[16] C. A. R. L. Brennand, et al., "An Intelligent Transportation System for Detection and Control of Congested Roads in Urban Centers," 2015 IEEE Symposium on Computers and Communication (ISCC), pp. 663-668, 2015.

[17] A. M. de Souza and L. A. Villas, "A Fully-distributed Traffic Management System to Improve the Overall Traffic Efficiency," Proceedings of the 19th ACM International Conference on Modeling, Analysis and Simulation of Wireless and Mobile Systems, pp. 19-26, 2016.

[18] A. M. de Souza, et al., "Traffic management systems: A classification, review, challenges, and future perspectives," International Journal of Distributed Sensor Networks, vol. 13, no. 4, pp. 1-14, 2017.

[19] R. Bauza, et al., "Road traffic congestion detection through cooperative Vehicle-to-Vehicle communications," IEEE Local Computer Networks Conference, pp. 606-612, 2010.

[20] G. B. Ara, et al., "CARTIM : A Proposal Toward Identification and Minimization of Vehicular Traffic Congestion for VANET,” 2014 IEEE Symposium on Computers and Communications (ISCC), pp. 1-6, 2014.

[21] L. Rui, et al., "A new traffic congestion detection and quantification method based on comprehensive fuzzy assessment in VANET," KSII Transactions on Internet and Information Systems, vol. 12, no. 1, pp. 41-60, 2018.

[22] R. Wang, et al., "V2V-based method for the detection of road traffic congestion," IET Intelligent Transport Systems, vol. 13, no. 5, pp. 880-885, 2019.

[23] E. Zhang and X. Zhang, "Road Traffic Congestion Detecting by VANETs," Proceedings of the 2nd International Conference on Electrical and Electronic Engineering, pp. 242-248, 2019.

[24] K. Z. Ghafoor, et al., "A fuzzy logic approach to beaconing for vehicular ad hoc networks," Telecommunication Systems, vol. 52, no. 1, pp. 139-149, 2013.

[25] Y. H. Wen and T. T. Lee, "Fuzzy data mining and grey recurrent neural network forecasting for traffic information systems," IRI-2005 IEEE International Conference on Information Reuse and Integration Conference, pp. 356-361, 2005.

[26] C. Wu, et al., "VANET broadcast protocol based on fuzzy logic and lightweight retransmission mechanism," IEICE Transactions on Communications, vol. 95-B, no. 2, pp. 415-425, 2012.

[27] L. Altoaimy and I. Mahgoub, "Fuzzy logic based localization for vehicular ad hoc networks," 2014 IEEE Symposium on Computational Intelligence in Vehicles and Transportation Systems, pp. 121-128, 2014.

[28] R. Meneguette, et al., "Enhancing Intelligence in Inter-vehicle Communications to Detect and Reduce Congestion in Urban Centers," The Twntiesth IEEE Symposium on Computers and Communications, pp. 662-667, 2015.

[29] R. I. Meneguette, et al., "Increasing Intelligence in Inter-Vehicle Communications to Reduce Traffic Congestions : Experiments in Urban and Highway Environments," PLoS ONE, vol. 11, no. 8, pp. 1-25, 2016.

[30] A. M. De Souza, et al., "ICARUS : Improvement of traffic Condition through an Alerting and Re-routing System," Computer Networks, vol. 110, pp. 118-132, 2016.

[31] M. A. Mondal and Z. Rehena, "Intelligent Traffic Congestion Classification System using Artificial Neural Network," International World Wide Web Conference Committee, pp. 110-116, 2019. 
[32] A. M. De Souza, et al., "SCORPION: A solution using cooperative rerouting to prevent congestion and improve traffic condition," 2015 IEEE International Conference on Computer and Information Technology;Ubiquitous Computing and Communications; Autonomic and Secure Computing; Pervasive Intelligence and Computing, pp. 497-503, 2015.

[33] A. M. De Souza, et al., "Real-time path planning to prevent traffic jam through an intelligent transportation system," 2016 IEEE Symposium on Computer and Communication (ISCC), pp. 726-731, 2016.

[34] J. O. Ensley, "Application of Highway Capacity Manual 2010 Level-of-Service Methodologies for Planning Deficiency Analysis," Master Theses, University of Tennessee, Knoxville, p. 162, 2012.

[35] R. Bauza and J. Gozalvez, "Traffic congestion detection in large-scale scenarios using vehicle-to-vehicle communications," Journal of Network and Computer Applications, vol. 36, no. 5, pp. 1295-1307, 2013.

[36] R. P. Roess, "Level of Service Concepts: Development, Philosophies, and Implications," Transportation Research Record Journal of the Transpotation Research Board, pp. 1-6, 1984.

[37] M. B. Younes and A. Boukerche, "Efficient traffic congestion detection protocol for next generation VANETs," IEEE International Conference on Communications (ICC), pp. 3764-3768, 2013.

[38] M. Gramaglia, et al., "ABEONA Monitored Traffic: VANET-Assisted Cooperative Traffic Congestion Forecasting," IEEE Vehicular Technology Magazine, vol. 9, pp. 50-57, 2014.

[39] M. Schönhof and D. Helbing, "Criticism of three-phase traffic theory," Transportation Research Part B Methodological, vol. 43, no. 7, pp. 784-797, 2009.

[40] S. B. Kotsiantis, et al., "Supervised Machine Learning: A Review of Classification Techniques," Informatica An International Journal of Computing and Informatics, vol. 31, no. 3, pp. 249-268, 2007.

[41] D. Zuev and A. W. Moore, "Traffic Classification Using a Statistical Approach," International Workshop on Passive and Active Network Measurement, pp. 321-324, 2005.

[42] A. Pathak and J. Vashistha, "Classification Rule and Exception Mining Using Nature Inspired Algorithms," International Journal of Computer Science and Information Technologies, vol. 6, no. 3, pp. 3023-3030, 2015.

[43] M. Becker, et al., "Traffic analysis and classification with bio-inspired and classical algorithms in sensor networks," 2008 International Symposium on Performance Evaluation of Computer Telecommunication Systems, pp. 67-73, 2008.

[44] N. I. Ramli and M. I. M. Rawi, "Hemorheology Based Traffic Congestion and Forecasting Model in the Internet of Vehicles," Asian Simulation Conference, pp. 365-379, 2017.

[45] Z. Sun, et al., "Traffic Congestion Forecasting Based on Possibility Theory," International Journal of Intelligent Transportation Systems Research, vol. 14, no. 2, pp. 85-91, 2016.

[46] X. Yu, et al., "Research on campus traffic congestion detection using BP neural network and Markov model," Journal of Information Security and Applications, vol. 31, pp. 54-60, 2016.

[47] H. Dezani, et al., "Controlling Traffic Jams on Urban Roads Modeled in Coloured Petri Net using Genetic Algorithm," IECON 2012 - 38th Annual Conference on IEEE Industrial Electronics Society, pp. 3043-3048, 2012.

[48] A. Dahiya, et al., "Vehicular Ad hoc Networks (VANETS): Simulation and Simulators," International Journal of Research in Management, Science and Technology, vol. 2, no. 1, pp. 2321-3264, 2014.

[49] D. Krajzewicz, et al., "Recent Development and Applications of SUMO - Simulation of Urban MObility," International Journal on Advances in Systems and Measurements, vol. 5, no. 3-4, pp. 128-138, 2012.

[50] "Documentation - Veins." Available: https://veins.car2x.org/documentation/.

\section{BIOGRAPHIES OF AUTHORS}
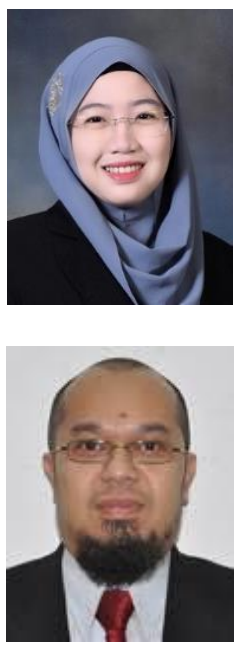

Nurshahrily Idura Ramli received the B.Sc. (Hons) in Data Communication and Networking from Universiti Teknologi MARA (UiTM), Malaysia. Currently she is also pursuing her Ph.D. studies while working in the same university as a senior lecturer in the Faculty of Computer and Mathematical Sciences. She obtained her M.Sc. degree in Computer Information Networks from the University of Essex, United Kingdom. She has been an IEEE member since 2015 and has more than 15 years of teaching and research experiences. Her current research interest includes Computer Network Communications, Vehicular Ad hoc Networks, Internet of Things, Wireless Sensor Networks as well as Technology Application in Education.

Mohd Izani Mohamed Rawi is currently a senior lecturer from the Faculty of Computer and Mathematical Sciences, Universiti Teknologi MARA (UiTM), Malaysia. He had his BSc. degree in Computer Science and Education from Universiti Teknologi Malaysia (UTM) and MSc. in Knowledge Based Systems from Oxford Brookes University, United Kingdom. $\mathrm{He}$ received his Ph.D. degree in Electronic Engineering (Wireless Sensor Network) from Aucland University of Technology, New Zealand and is a member of IEEE since 2015. His current research interests include wireless ad hoc and sensor networks, wireless networks, mobile and pervasive computing, Internet of Things (IoT) and embedded intelligence. 